\title{
Seipin mutation at glycosylation sites activates autophagy in transfected cells via abnormal large lipid droplets generation
}

\author{
Hua-dong FAN ${ }^{1,2}$, Shao-peng $\mathrm{CHEN}^{2}$, Yu-xiang SUN² , Shao-hai XU ${ }^{1,2}$, Li-jun WU $\mathrm{WU}^{1,2, *}$ \\ ${ }^{1}$ School of Nuclear Science and Technology, University of Science and Technology of China, Hefei 230026, China; ${ }^{2}$ Key Laboratory of \\ Ion Beam Bioengineering, Chinese Academy of Sciences and Anhui Province, Hefei 230031, China
}

\begin{abstract}
Aim: Seipin is a protein that resides in endoplasmic reticulum, and involved in both lipid metabolic disorders and motor neuropathy. The aim of this study was to investigate the effects of mutant seipin on autophagy system and the morphology of lipid droplets in vitro. Methods: HEK-293, H1299 and MES23.5 cells were transfected with the plasmids of mutated seipin at glycosylation sites (N88S or S9OL) and GFP-LC3 plasmids. The cells were subjected to immunofluorescence and flow cytometry assays, and the cell lysates were subjected to immunoblot analysis. Nile Red was used to stain the lipid droplets in the cells.

Results: Overexpression of the mutated seipin proteins N88S or S90L activated autophagy in the 3 cell lines, and substantially altered the sub-cellular distribution of the autophagosome marker GFP-LC3, leading to a number of large vacuoles appearing in the cytoplasm. The sub-cellular location of GFP-LC3 and mutated seipin proteins highly overlapped. Moreover, and the mutated seipin proteins caused diffuse small lipid droplets to fuse into larger lipid droplets. Treatment of mutated seipin-transfected cells with the autophagy inhibitor 3-MA ( $5 \mathrm{mmol} / \mathrm{L}$ ) facilitated the fusion of mutated seipin-induced large vacuoles. The protein glycosylation inhibitor tunicamycin could mimic the mutated seipin-induced effects, and treatment of the wild-type seipin-transfected cells with tunicamycin ( $2.5 \mu \mathrm{g} / \mathrm{mL}$ ) produced similar morphological and biochemical properties as in the mutated seipin-transfected cells.

Conclusion: The mutation of seipin at glycosylation sites disrupt its function in regulating lipid droplet metabolism, and the autophagy acts as an adaptive response to break down abnormal lipid droplets. The interruption of autophagy would accelerate the fusion of abnormal lipid droplets.
\end{abstract}

Keywords: seipin; lipodystrophy; motor neuron disease; glycosylation; lipid droplets; autophagy; 3-MA; tunicamycin; endoplasmic reticulum

Acta Pharmacologica Sinica (2015) 36: 497-506; doi: 10.1038/aps.2014.164; published online 23 Mar 2015

\section{Introduction}

The seipin/bscl2 gene was first linked to the metabolic disorder Berardinelli-Seipin congenital lipodystrophy 2 (BSCL2) by Magre in 2001. BSCL2 is an autosomal-recessive disease characterized by severe lipoatrophy, insulin resistance, and hypertriglyceridemia, which are all correlated with dysfunctional lipid metabolism ${ }^{[1-4]}$. After its identification, seipin/bscl2 was also found to be related to the autosomal dominant motor neuron disease distal Hereditary Motor Neuropathy (dHMN), which is characterized by the nearly exclusive degeneration of motor nerve fibers predominantly in the distal part of $\operatorname{limbs}^{[4,5]}$. Genetic and proteomic analyses revealed that the missense mutation A212P and the mutations N88S and S90L

\footnotetext{
* To whom correspondence should be addressed.

E-mail ljw@ipp.ac.cn

Received 2014-10-25 Accepted 2014-12-29
}

that disrupt N-glycosylation are involved in the pathogenesis of BSCL2 and dHMN, respectively ${ }^{[1,4]}$.

Human seipin is a protein in the endoplasmic reticulum (ER) that possesses an N-glycosylation site facing the ER lumen with a conservative region (amino acid residues 31-252) between its two transmembrane domains ${ }^{[1]}$. It has been reported that the $\mathrm{dHMN}$-related seipin mutants $\mathrm{N} 88 \mathrm{~S}$ and S90L, which are misfolded in the ER, can trigger ER stress and therefore enhance the ubiquitination and degradation of these mutants due to their lack of glycosylation ${ }^{[6]}$. Moreover, the missense mutation in A212P's conservative region has been associated with lipodystrophy coupled with insulin resistance and mental retardation ${ }^{[1]}$.

Lipid droplets (LDs) are emerging organelles crucial to lipid metabolism-related diseases ${ }^{[7,8]}$. LDs consist of a core of neutral lipids, predominantly triacylglycerols (TAGs) or sterol esters (SEs), surrounded by a monolayer of phospholipids 
and associated proteins ${ }^{[9]}$. LDs are believed to originate from the microdomains of the endoplasmic reticulum, where the enzymes for the synthesis of neutral lipids reside ${ }^{[9,10]}$. The intracellular storage and utilization of lipids are crucial for maintaining cellular energy homeostasis ${ }^{[11]}$, and LDs are the cellular storage sites of neutral lipids ${ }^{[12]}$. Under lipid overload conditions, the quantity of small LDs increases, and the small LDs cluster together to form large $\operatorname{LDs}^{[7,12]}$. Obesity and lipodystrophy are characterized by an accumulation or loss of body fat, respectively, and both conditions produce lipid storage in non-adipose tissues, leading to lipotoxicity ${ }^{[8]}$.

Seipin is a transmembrane protein that resides in the ER and is concentrated at the junctions between the ER and cytosolic $\mathrm{LDs}^{[13]}$. Seipin is one of the most important factors regulating lipid metabolism ${ }^{[12]}$. Previous studies have demonstrated that human seipin participates in the regulation of LD size in yeasts $^{[14]}$, the knockdown of seipin significantly increases TAG levels and the clustering of small LDs ${ }^{[15]}$, and the loss of seipin function disturbs adipocyte differentiation ${ }^{[2,15]}$. In addition, there is evidence that the autophagy system participates in the metabolism of LDs and the autophagy pathway components associated with $\operatorname{LDs}^{[11,16-19]}$. For example, the inhibition of autophagy in cultured hepatocytes and mouse liver cells increases triglyceride storage in LDs ${ }^{[11]}$, and the glycosylationsite mutated seipins N88S and S90L tend to form inclusion bodies within the ER, triggering ER stress and subsequent cell apoptosis $^{[1,4,5,20]}$.

To date, however, there have been no reports showing the relationship between human seipin, LDs and the autophagy system. In the present study, we investigated the relationship of mutant seipin to the autophagy system and the morphology of LDs.

\section{Materials and methods}

\section{Plasmid constructs and reagents}

PCS2+MT-myc-seipin (wt), PCS2+MT-myc-seipin (N88S), and PCS2+MT-myc-seipin (S90L) were kind gifts from Professor Daisuke ITO at Keio University (Japan). GFP-LC3 and the empty vector PCS2+MT were provided by Professor Guanghui WANG at SooChow University (China). All of the constructs were confirmed by sequencing. Tunicamycin, 3-methyladenine (3-MA), and saponin were purchased from Sigma.

\section{Cell cultures and transfection}

HEK-293, H1299, and MES23.5 cells were cultured in Dulbecco's modified Eagle's medium (DMEM, GIBCO) containing 10\% fetal bovine serum (FBS, Hyclone). The cells were transfected with expression vectors using the TurboFect Transfection Reagent (Thermo Scientific, Waltham, MA, USA) in DMEM with 10\% FBS at approximately $40 \%$ confluence according to the manufacturer's instructions.

\section{Immunocytochemistry and Nile Red dye}

The HEK-293 cells were grown and transfected into 24-well cell dishes, washed with PBS and fixed with $4 \%$ paraformaldehyde for $5 \mathrm{~min}$ at room temperature, treated with $0.25 \%$ Triton
X-100 for 15 min, and subsequently blocked with 4\% FBS for $1 \mathrm{~h}$ at room temperature. Next, the cells were incubated with a primary antibody overnight at $4{ }^{\circ} \mathrm{C}$, followed by incubation with a Rho-conjugated donkey anti-mouse secondary antibody (Santa Cruz Biotechnology, Santa Cruz, CA, USA). The nuclei were stained with DAPI (Sigma, St Louis, MO, USA).

A stock solution of Nile Red $(1000 \mu \mathrm{g} / \mathrm{mL}$, Life Technologies, USA) in acetone was prepared and stored in darkness. The transfected HEK-293 cells were fixed with 1.5\% glutaraldehyde in PBS for $10 \mathrm{~min}$, and then the dye was added directly at dilution of 1:1000. After a 10-min incubation at room temperature, the excess dye was removed with a brief rinsing in PBS.

\section{Immunoblot analyses and antibodies}

The cells were lysed in a buffer containing $50 \mathrm{mmol} / \mathrm{L}$ Tris$\mathrm{HCl}$ (pH 7.6), $150 \mathrm{mmol} / \mathrm{L} \mathrm{NaCl}, 0.5 \%$ sodium deoxycholate, $1 \%$ Nonidet P-40 and protease inhibitor (Roche). The proteins were then separated by 10\%-12\% SDS-PAGE and transferred to a PVDF membrane (Millipore, Billerica, MA, USA).

The primary antibodies used were mouse anti-myc (Sigma, St Louis, MO, USA), mouse anti-Bip (Zen Bioscience, Chengdu, China), mouse anti-actin (TransGen Biotech, Beijing, China), and rabbit anti-LC3 (Cell Signaling, Danvers, MA, USA). The secondary antibodies used were sheep anti-mouse IgG-HRP and anti-rabbit IgG-HRP (Amersham Bioscience, Piscataway, NJ, USA). The proteins were visualized using an ECL detection kit (Amersham Bioscience, Piscataway, NJ, USA).

\section{Flow cytometry tests}

The GFP-LC3 transfected cells were harvested with trypsin and washed with PBS with or without $0.05 \%$ saponin. Over 10000 events were captured for each analysis. The FACS data were collected using a BD FACSCalibur flow cytometer, and the data analyses were carried out with FlowJo 7.6 software.

\section{Statistical analysis}

The densitometric values of the Western blot bands of three independent experiments were analyzed by Photoshop 7.0. The data were analyzed with one-way analysis of variance (ANOVA) using Origin 7.0 software (Originlab, USA).

\section{Results}

\section{Co-localization of seipin and LC3}

It has been reported that seipin can regulate the size of LDs as well as control the fusion of lipid droplets in yeasts ${ }^{[14,21]}$. Additionally, researchers have recently found that autophagy and lipid metabolism are closely related in eukaryotic cells deprived of nutrients ${ }^{[1,16]}$, and one of the autophagy components, LC3, is involved in the formation of lipid droplets ${ }^{[17,18]}$. To investigate whether seipin is directly related to the autophagy system, we co-transfected wild-type seipin and two seipin mutants with the autophagosome marker GFP-LC3 into HEK293 cells. As shown in Figure 1, GFP-LC3 only partly co-localized with wild-type seipin in the cytoplasm, whereas GFP-LC3 

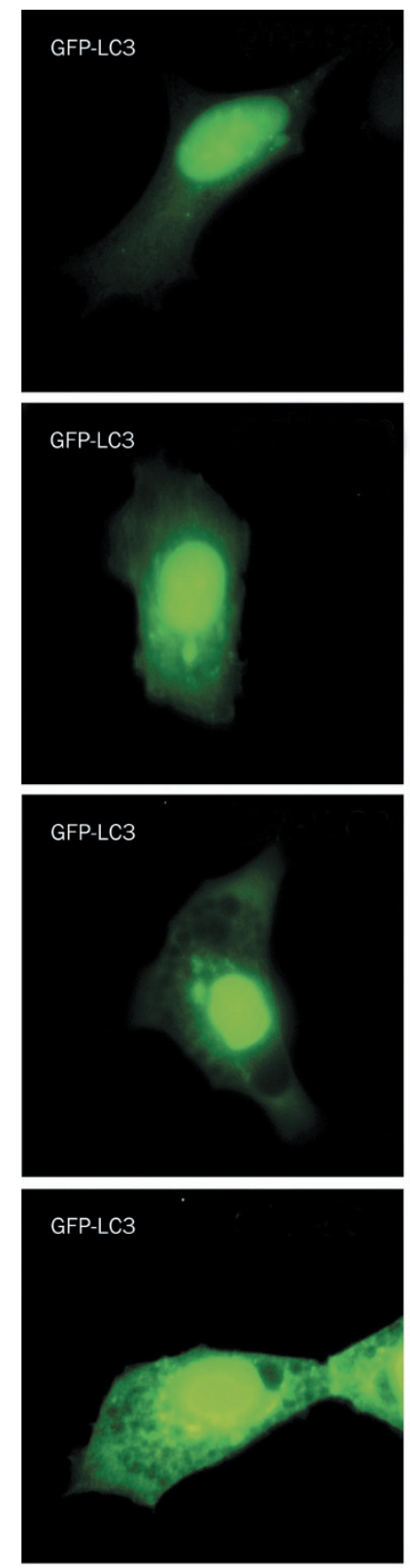
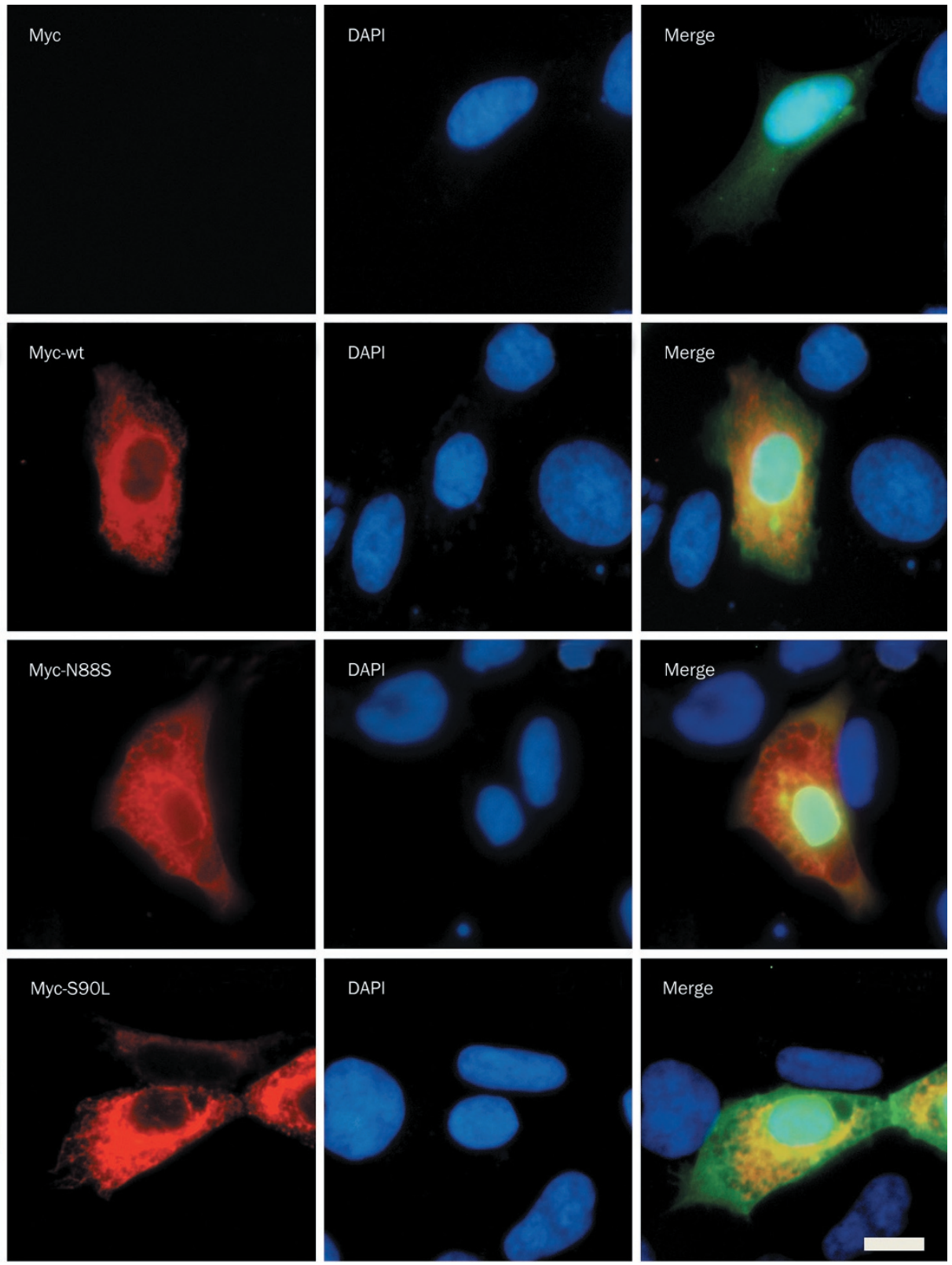

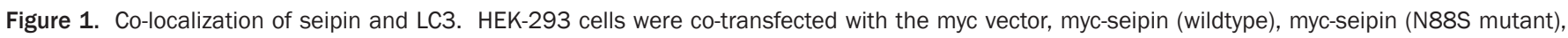

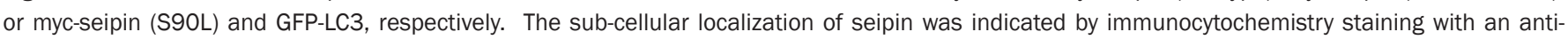

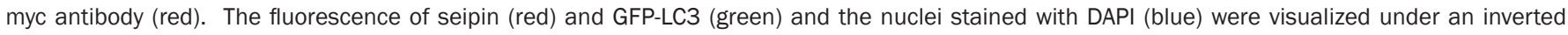
microscope (Olympus IX71). The scale bar is $10 \mu \mathrm{m}$.

and mutant seipin (N88S or S90L) were highly co-localized in the cytoplasm. Moreover, vacuoles enveloped in GFP-LC3 or seipin appeared in the cytoplasm of mutant seipin-transfected cells but not wild-type seipin-transfected cells.

Overexpression of the seipin mutants (N88S or S90L) altered the pattern of the sub-cellular distribution of the autophagosome marker LC3

To further investigate whether the vacuoles in the mutant seipin-transfected cells was cell line specific, co-transfection experiments were performed in three different cell lines. As shown in Figure 2A, GFP-LC3 was uniformly expressed in HEK-293 cells transfected solely with the empty vector Myc or with Myc-seipin (wt). On the contrary, cells transfected with mutant seipin (seipin N88S and S90L) exhibited giant vacuoles in the cytoplasm that were bordered by GFP-LC3. This phenomenon was repeated in the H1299 and MES23.5 dopaminergic cell lines (Figure 2C and 2E). These results imply that the mutations at the $\mathrm{N}$-glycosylation site of seipin altered the subcellular distribution of the autophagosome marker LC3. 
A
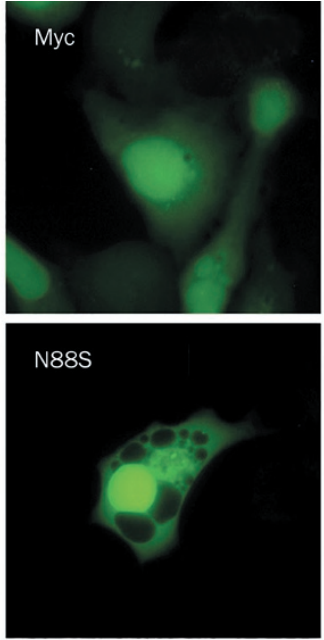

C
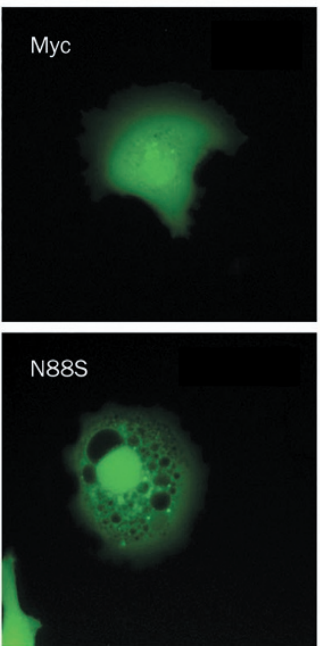

E
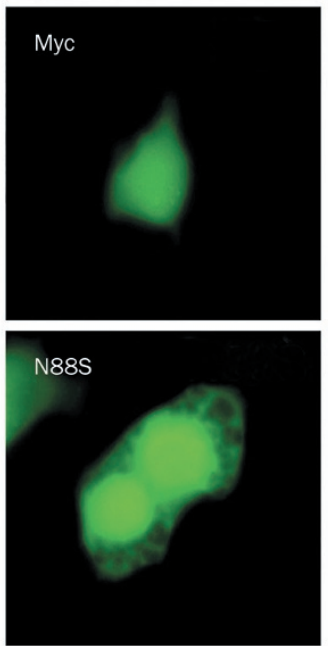
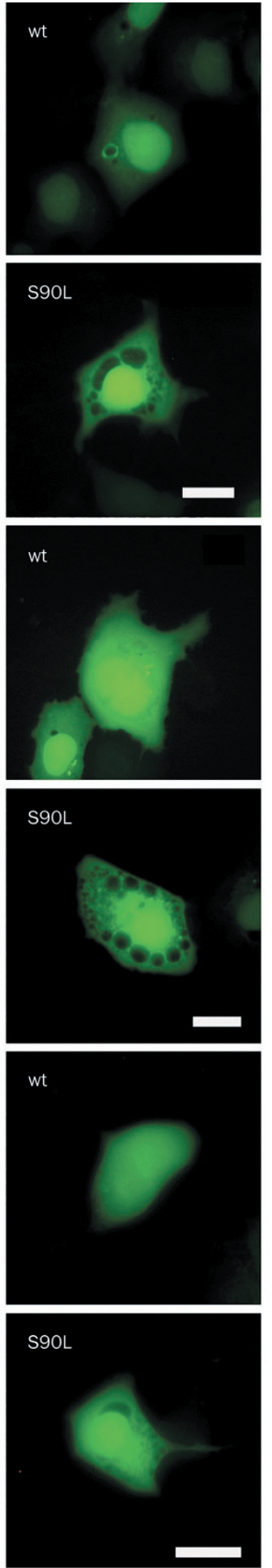

B

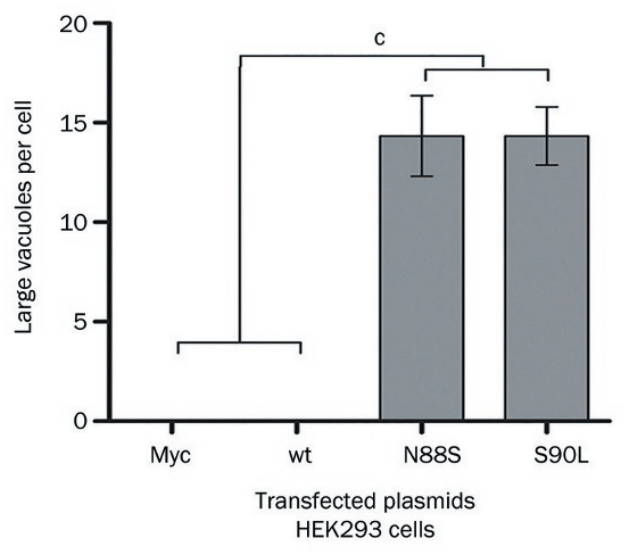

D

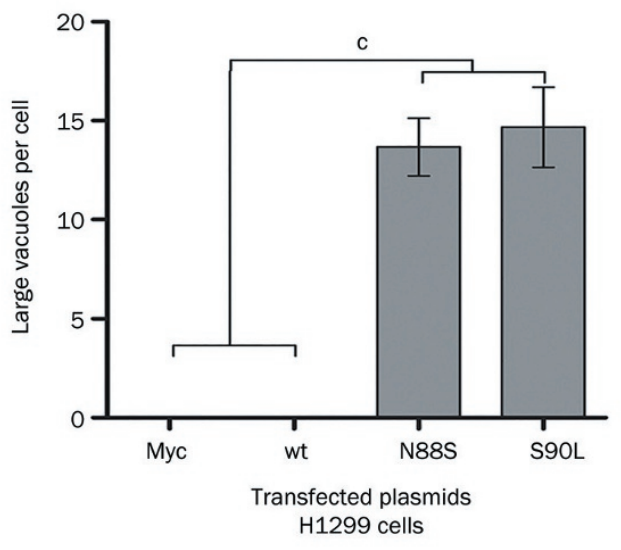

F

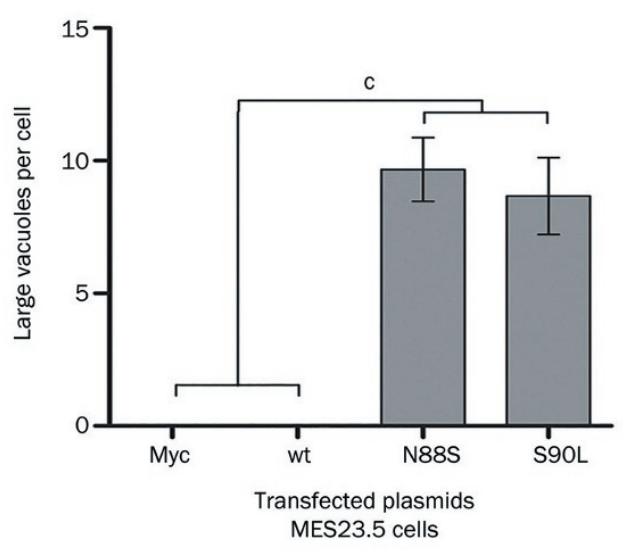

Figure 2. The sub-cellular distribution of the autophagic marker GFP-LC3 is influenced by the overexpression of seipin mutants. (A) HEK-293 cells were co-transfected with the Myc vector, myc-seipin (wild-type), myc-seipin (N88S mutant), or myc (S90L mutant) and GFP-LC3, respectively. After transfection for $24 \mathrm{~h}$, the cells were observed under an inverted microscope (Olympus IX71). (C) The H1299 cells and (E) MES23.5 cells were treated using the same method used in (A). The scale bars are $10 \mu \mathrm{m}$. The quantitative data from three independent experiments is shown in (B, D, and F) and are represented as the mean \pm SEM of the large vacuoles per cell. ${ }^{\mathrm{C}} P<0.01$. 
A
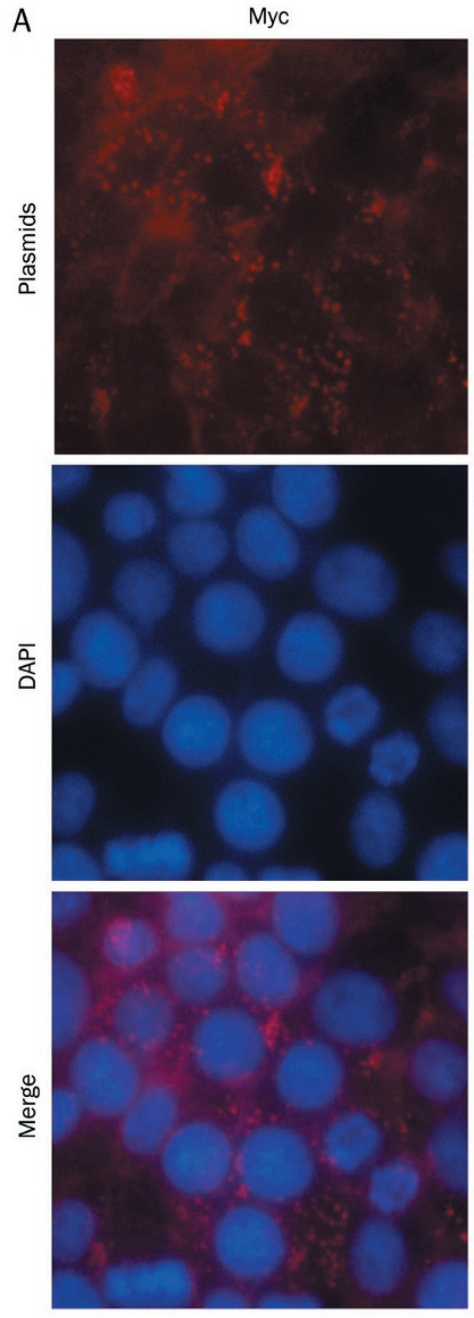

B wt
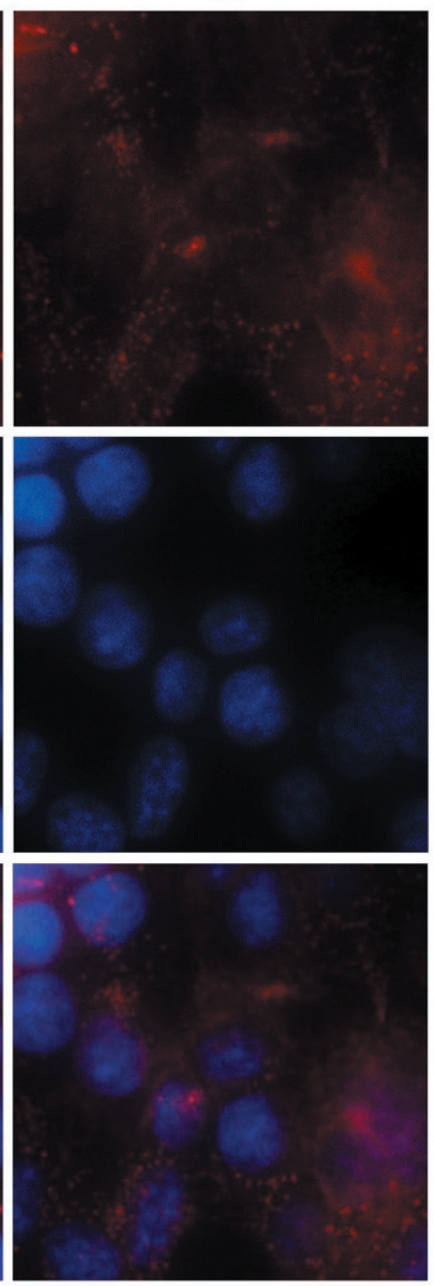

N88S
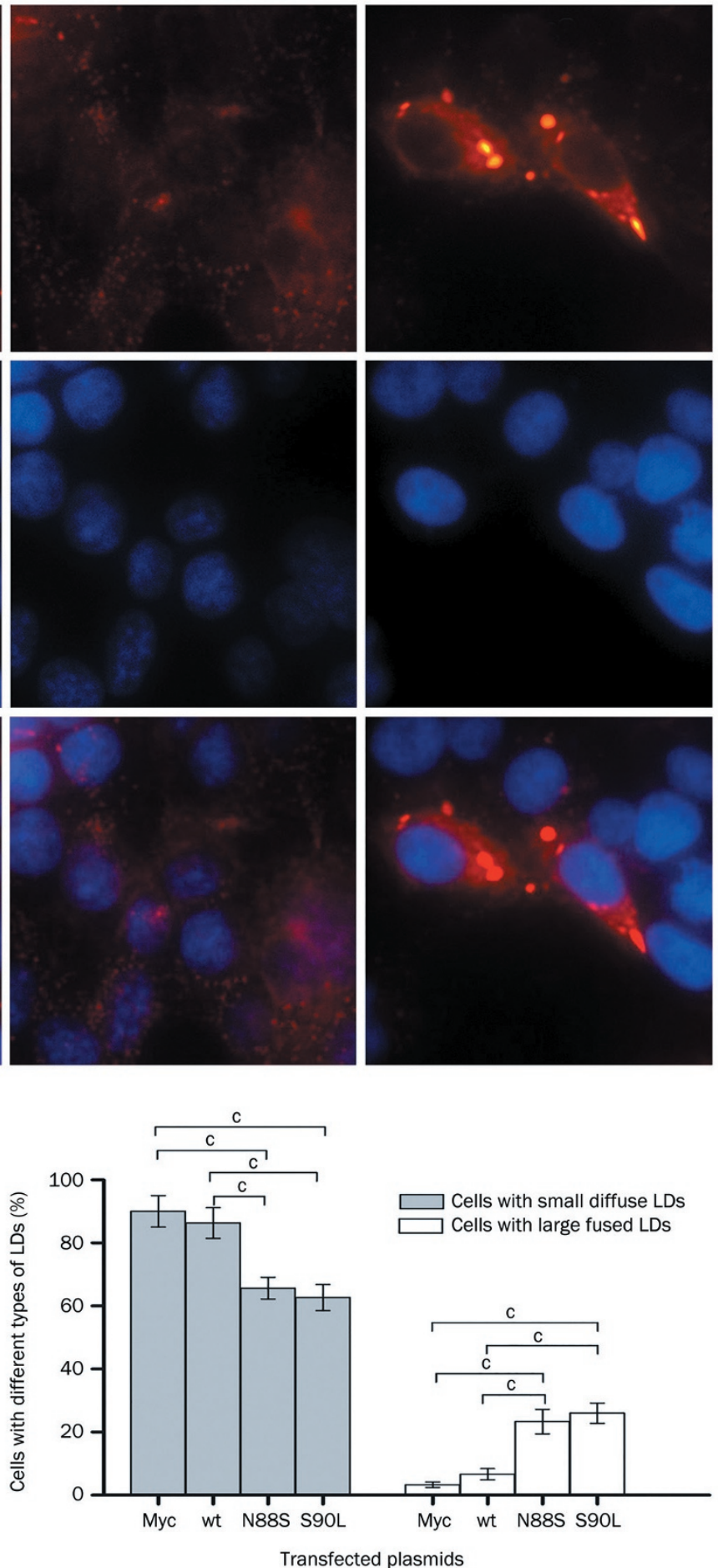

Transfected plasmids
S90L
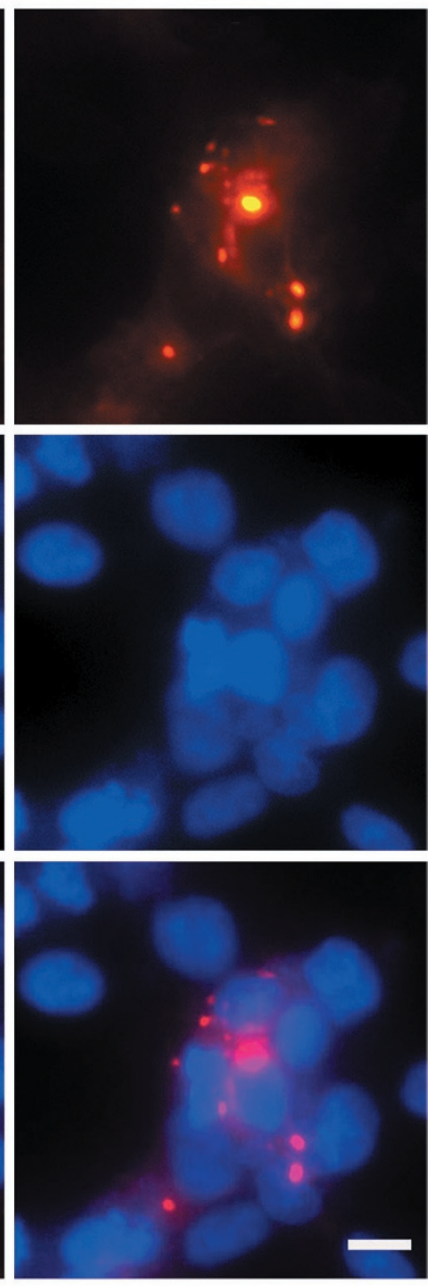

Figure 3. Mutant seipin influences the morphology of LDs. (A) HEK-293 cells were transfected with the myc vector, myc-seipin (wildtype), myc-seipin (N88S) or myc-seipin (S90L). The cells were fixed and stained with Nile Red, and the nuclei were stained with DAPI (blue) $24 \mathrm{~h}$ after transfection and then visualized under an inverted microscope (Olympus IX71). The scale bar is $10 \mu \mathrm{m}$. (B) The percentage of cells with small diffuse LDs or large fused LDs was quantified from three independent experiments and is shown as the mean \pm SEM. ${ }^{c} P<0.01$. 
A
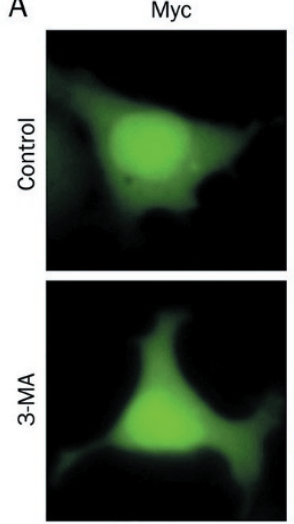

Myc-wt
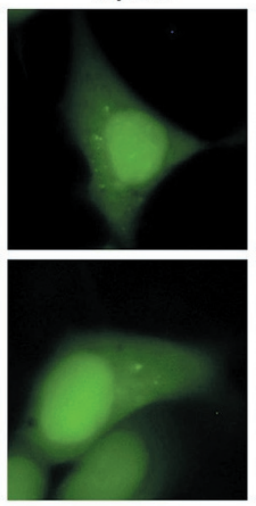

Myc-N88S
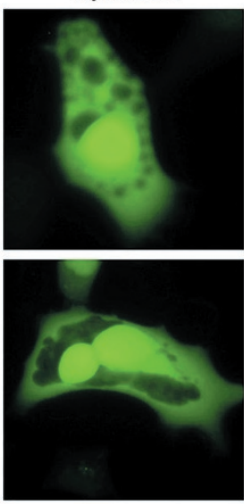

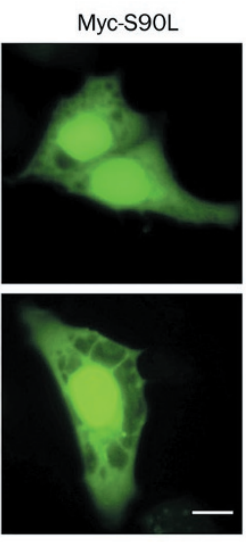

B

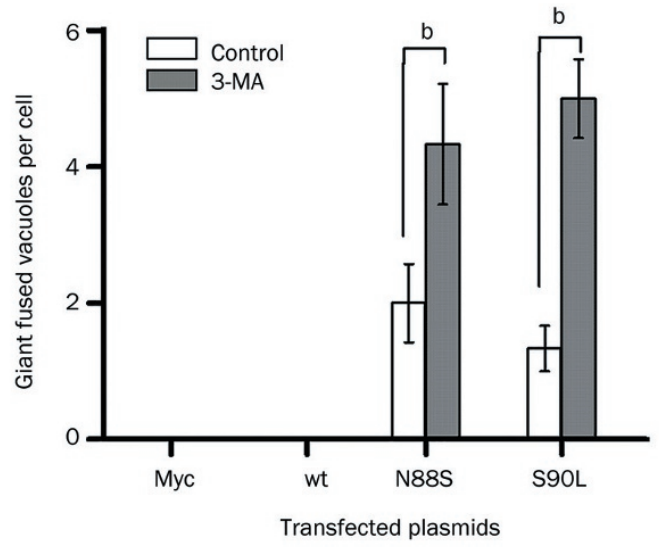

D
C

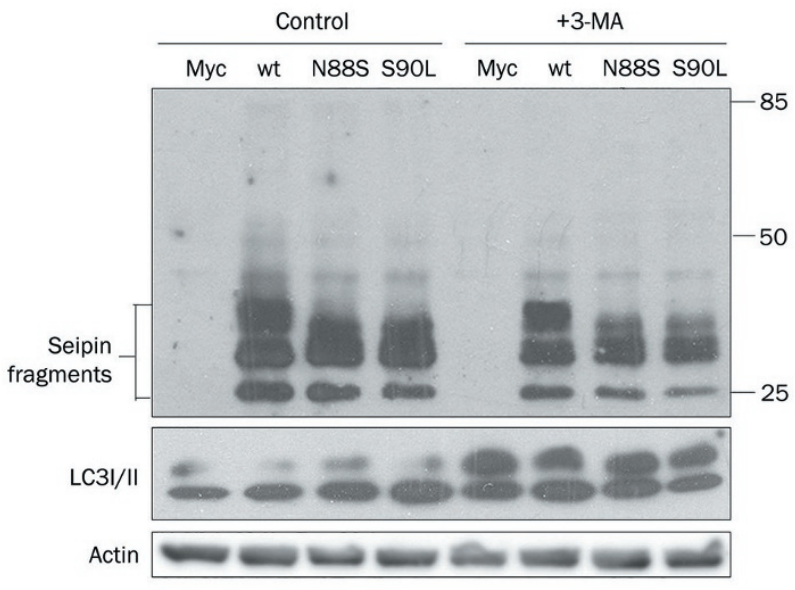

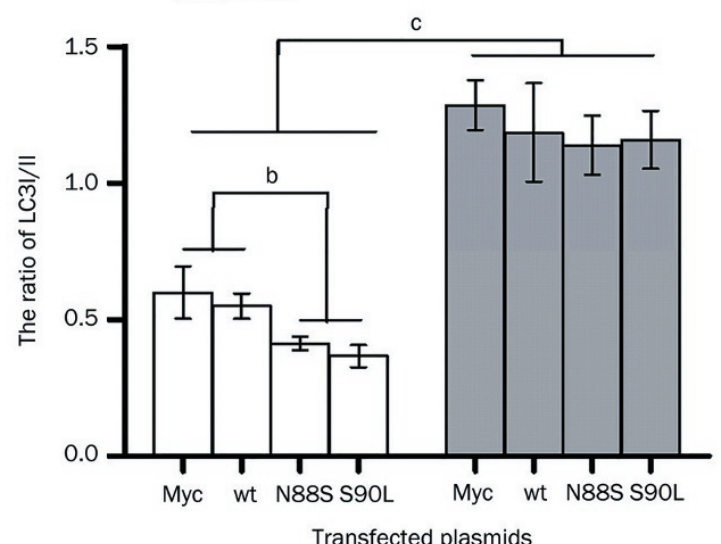

Figure 4. The inhibition of autophagy with 3-MA facilitated the fusion of mutant seipin-induced abnormal vacuoles. (A) HEK-293 cells were transfected with either the myc, myc-wt, myc-N88S or myc-S90L plasmid with or without the autophagy inhibitor 3-MA (5 mmol/L). The fluorescence of GFP-LC3 was visualized under an inverted microscope (Olympus IX71) $24 \mathrm{~h}$ after transfection. The scale bar is $10 \mu \mathrm{m}$. (B) The quantitative data of three independent experiments is indicated as the mean \pm SEM. ${ }^{\mathrm{b}} \mathrm{P}<0.05$. (C) HEK-293 cells were transfected with either the myc, myc-wt, myc-N88S or myc-S90L plasmid with or without 3-MA $(5 \mathrm{mmol} / \mathrm{L})$. The cells were harvested $24 \mathrm{~h}$ after transfection and lysed for Western blots using the primary antibodies anti-myc, anti-LC3 and anti-actin. (D) The quantitative analysis of the ratio of LC3I to LC3II from three independent experiments is indicated as the mean \pm SEM. ${ }^{\mathrm{b}} P<0.05,{ }^{\mathrm{c}} \mathrm{P}<0.01$.

\section{Mutant seipin affected the morphology of LDs}

Because human seipin has been reported to regulate the size of LDs in yeasts ${ }^{[14]}$, we were interested in finding out whether the two seipin mutants, N88S and S90L, directly impact the size of LDs in mammalian cells. It has been reported that LDs are remarkably diverse in size: a giant (>100 $\mu \mathrm{m}$ in diameter) unilocular LD occupies the entire cytoplasm of white adipocytes, whereas much smaller LDs with diameters less than $0.4 \mu \mathrm{m}$ are found in normal yeast cells ${ }^{[12]}$. In this study, we defined large fused LDs as having diameters greater than $1 \mu \mathrm{m}$ and small diffuse LDs as having diameters less than $0.4 \mu \mathrm{m}$. As shown in Figure 3A, the LDs stained with Nile Red became larger in the mutant seipin-transfected HEK-293 cells compared with the LDs in the empty vector myc or the wild-type seipin-transfected cells. The ratios of small diffuse LDs were $90 \%, 86.3 \%, 65.7 \%$, and $62.7 \%$ in the HEK-293 cells transfected with the myc vector, wild-type seipin, the N88S mutant and the S90L mutant, respectively. The ratios of large fused LDs were $3.3 \%, 6.7 \%, 23.3 \%$, and $26 \%$ in the HEK-293 cells transfected with the myc vector, wildtype seipin, the N88S mutant and the S90L mutant, respectively (Figure 3B).

Inhibition of autophagy with 3-MA facilitated the fusion of mutant seipin-induced abnormal vacuoles

To explore the role of autophagy in vacuole formation and its potential effects on LD morphology, the autophagy inhibitor 3-MA was used to interrupt the activation of autophagy before the transfection of the HEK-293 cells with wild-type seipin or mutant seipin plasmids. As shown in Figure 4A, in the 3-MA-treated group of mutant seipin-transfected cells, GFPLC3-bounded vacuoles significantly increased in diameter but decreased in quantity compared with the control group. Specifically, the mutant seipin-induced abnormal vacuoles became more largely fused, and autophagy was inhibited, whereas the 
cells transfected with the plasmids of myc or wild-type seipin exhibited no such effects. Figure $4 \mathrm{C}$ and $4 \mathrm{D}$ show that in the control group, the mutant seipins (N88S and S90L) activated autophagy, manifested by a decreased ratio of LC3I to LC3II; however, in the 3-MA-treated group, autophagy was inhibited compared with the control group, which was indicated by an increased ratio of LC3I to LC3II.

The protein glycosylation-inhibition drug tunicamycin can mimic the effects of mutant seipin

To further investigate whether the morphological changes in the GFP-LC3-monitored autophagy system and the LDs were due to the point mutations that disrupted the normal glycosylation of seipin, HEK-293 cells were treated with tunicamycin $(2.5 \mu \mathrm{g} / \mathrm{mL})$ for $8 \mathrm{~h}$ after being transfected with the Myc vector, Myc-seipin (wt), Myc-seipin (N88S) and Myc-seipin (S90L). As shown in Figure 5A, the tunicamycin-treated Mycseipin (wt)-transfected cells exhibited approximately the same effects as the seipin mutant-transfected cells, whereas the tunicamycin-treated myc vector-transfected cells had no such effects, suggesting that the tunicamycin-mediated morphological changes in the sub-cellular distribution of GFP-LC3 was seipin-dependent. As shown in Figure 5B, there was a significant increase in the number of large vacuoles in the cells
A

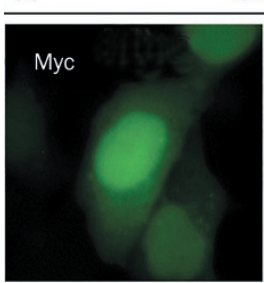

+DMSO
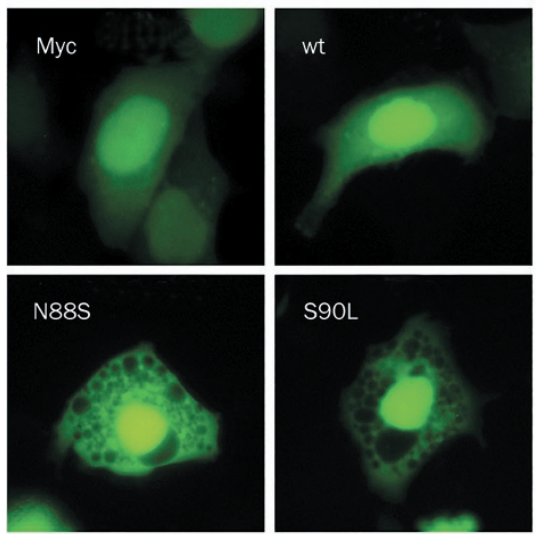

+Tunicamycin
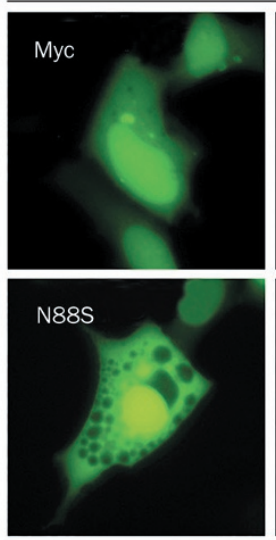
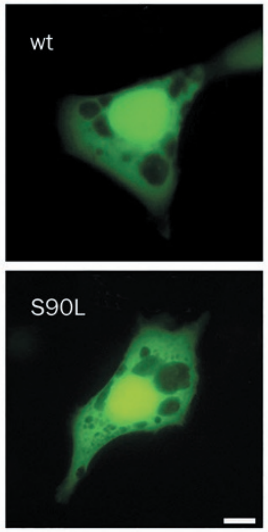

B

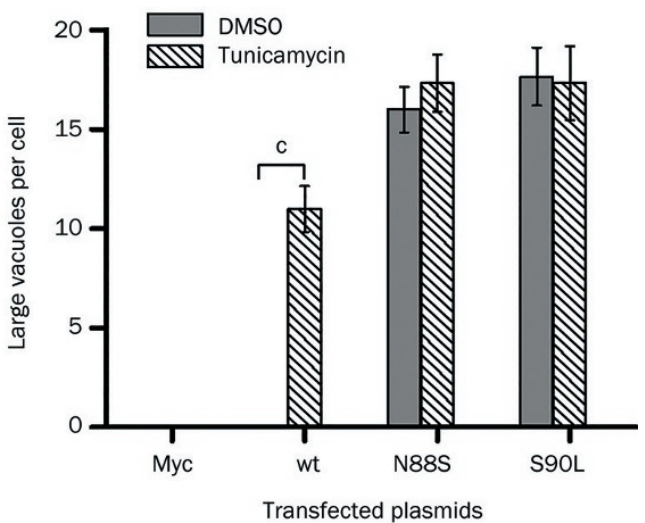

C

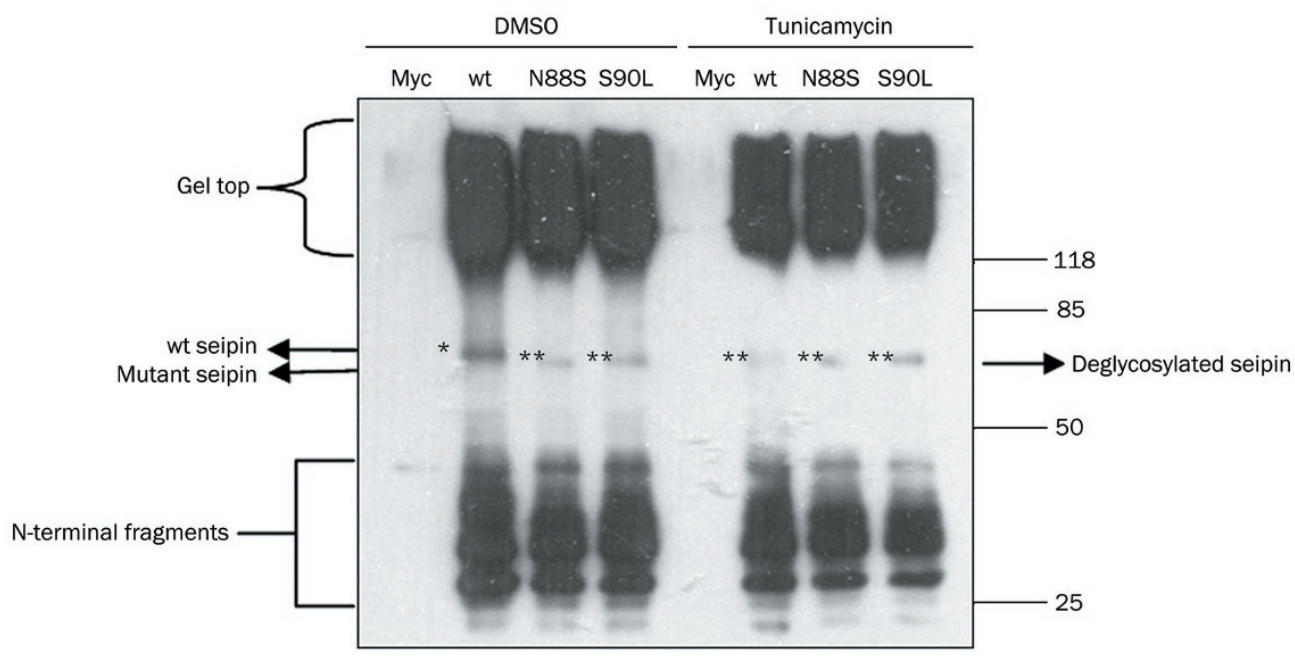

Figure 5. The protein glycosylation-inhibition drug tunicamycin mimics the effects of mutant seipin on the cytoplasmic redistribution of GFP-LC3 in HEK293 cells. (A) The HEK-293 control group cells were co-transfected with either the myc, myc-seipin (N88S), or myc-seipin (S90L) vector and GFP-LC3 in the presence of DMSO, and a second sample of HEK-293 cells was co-transfected with either the myc, myc-seipin (N88S), or myc-seipin (S90L) vector and GFP-LC3 in the presence of tunicamycin $(2.5 \mu \mathrm{g} / \mathrm{mL})$. The fluorescence of GFP-LC3 was visualized under an inverted microscope (Olympus IX71). The scale bar is $10 \mu \mathrm{m}$. (B) The quantitative data of three independent experiments is indicated as the mean \pm SEM. ${ }^{\circ} P<0.01$. (C) Western blot analysis of the glycosylated and deglycosylated forms of seipin. The HEK-293 cells of both groups were transfected with the myc vector, myc-seipin (wildtype), myc-seipin (N88S) or myc-seipin (S9OL) with or without tunicamycin $(2.5 \mu \mathrm{g} / \mathrm{mL})$. The cells were harvested $24 \mathrm{~h}$ after transfection, lysed, and detected on Western blots with an anti-myc primary antibody. Left panel: the single asterisk shows the full length molecular band of wildtype seipin, while the double asterisk shows the full length molecular bands of the two seipin mutants N88S and S9OL. The band of wildtype seipin is higher than the two seipin mutants due to glycosylation. Right panel: in the presence of tunicamycin $(2.5 \mu \mathrm{g} / \mathrm{mL})$, the full length molecular band of wildtype seipin as well as the pattern of its cleaved N-terminal fragments was identical to the seipin mutants N88S and S90L. The experiment was performed in triplicate and yielded similar results each time. 
that were transfected with wild-type seipin pre-treated with tunicamycin.

The inhibition of protein glycosylation by tunicamycin is also demonstrated by the Western blot in Figure 5C, which shows that the band consisting of full length Myc-seipin (wt) (marked with a single asterisk) was in a slightly higher posi- tion than the bands of the two seipin mutants [Myc-seipin (N88S) and Myc-seipin (S90L)] (marked with double asterisks). However, after being treated with tunicamycin, the band of full length Myc-seipin (wt) was lowered to the same position as the seipin mutants. Moreover, as shown in Figure 6A, in the DMSO-treated control group, Myc-seipin (wt) showed
A

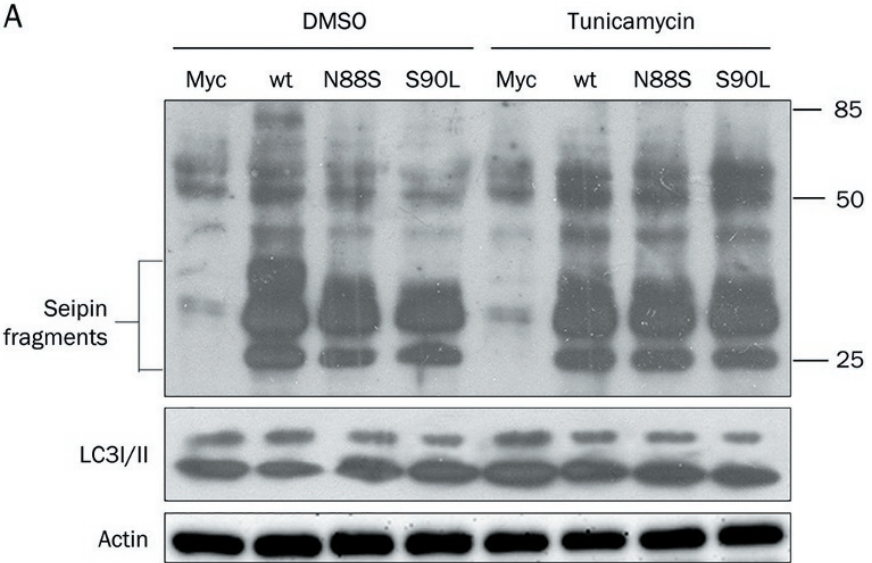

C

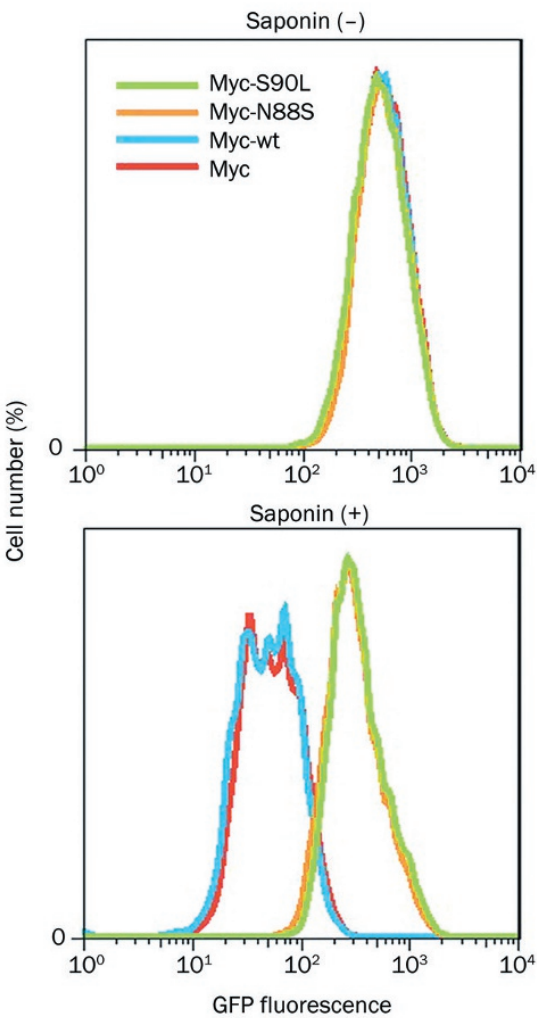

B

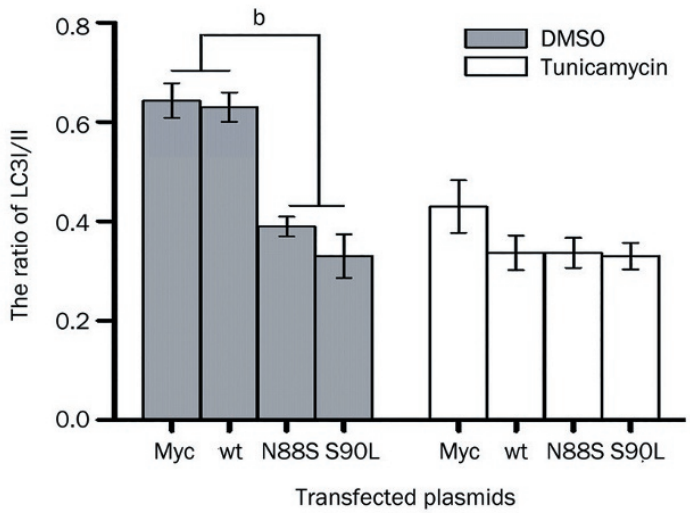

D
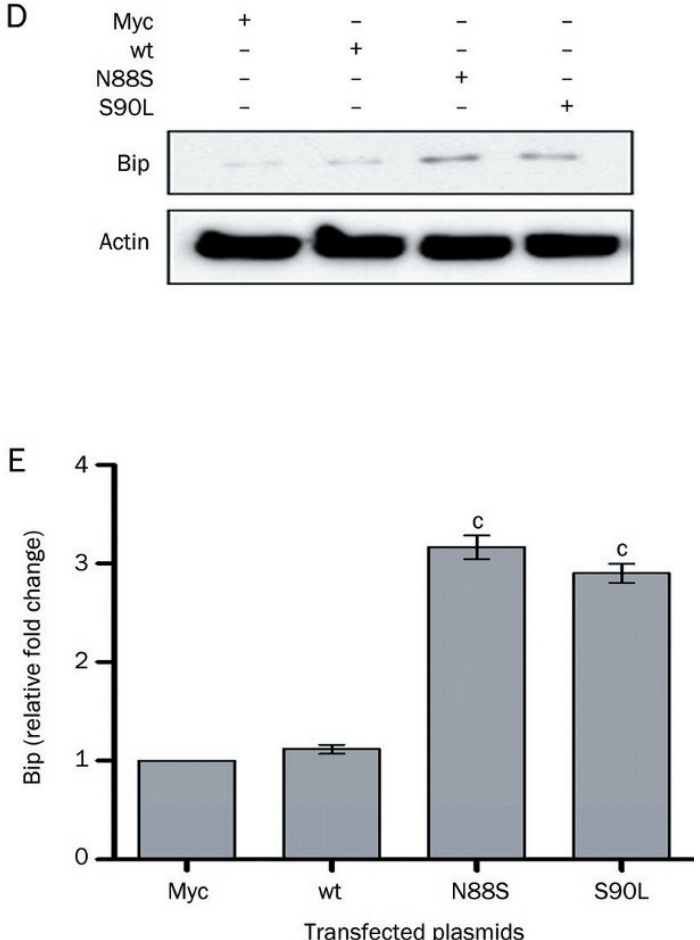

Figure 6. The autophagy system was induced by the overexpression of the seipin mutants N88S and S90L. (A) Left panel: HEK-293 cells were transiently transfected with either the myc, myc-seipin (wildtype), myc-seipin (N88S) or myc-seipin (S90L) vector in the presence of DMSO. Right panel: HEK-293 cells were transiently transfected with either the myc, myc-seipin (wildtype), myc-seipin (N88S) or myc-seipin (S9OL) vector in the presence of tunicamycin $(2.5 \mu \mathrm{g} / \mathrm{mL})$. (B) The quantitative analysis of the relative intensity ratio of LC3 I/II is shown as the mean \pm SEM from 3 independent experiments. ${ }^{\mathrm{b}} \mathrm{P}<0.05$. (C) HEK-293 cells were transfected with either myc, myc-seipin (wt), myc-seipin (N88S) or myc-seipin (S90L). After $24 \mathrm{~h}$ of transfection, GFP fluorescence was measured by FACS either with (bottom) or without (top) a wash containing $0.05 \%$ saponin. (D) HEK-293 cells were transfected with the myc vector, myc-seipin (wildtype), myc-seipin (N88S) or myc- seipin (S90L). The cells were harvested $24 \mathrm{~h}$ after transfection and lysed, and protein expression was detected with Western blots using anti-Bip and anti-actin primary antibodies. (E) The quantitative analysis of the Bip fold change is indicated as the mean \pm SEM from three independent experiments. ${ }^{c} P<0.01$. 
differently cleaved N-terminal fragments compared with the two seipin mutants (N88S and S90L), but in the tunicamycintreated group, Myc-seipin (wt) shared the same pattern of N-terminal fragments with the two seipin mutants, suggesting that the deglycosylation of the mutant seipins played a pivotal role in the seipin-induced morphological changes in the distribution pattern of GFP-LC3.

\section{Mutant seipins induce autophagy in a deglycosylation-dependent manner}

It has been reported that mutant seipin can form protein inclusions in the endoplasmic reticulum and induce ER stress ${ }^{[6]}$. As shown in Figure 6D and 6E, mutant seipin upregulated the ER stress indicator Bip while the LC3I/II ratio decreased in the mutant seipin groups (Figure 6A and 6B). When treated with tunicamycin, all of the LC3I/II ratios decreased compared with each non-drug-treated group (Figure 6A and 6B). Furthermore, saponin was used to remove soluble GFP-LC3I in the cytoplasm. As shown in Figure 6C, which is consistent with the Western blot in Figure 6A, the GFP fluorescence of the seipin mutant-transfected cells was higher than the wild-type seipin-transfected cells and control group, whereas the total GFP fluorescence was uniform across all four groups without saponin, indicating that the autophagy system was indeed activated after the transfection of the seipin mutants. These results are also consistent with the morphological changes of the LDs mediated by the autophagy component GFP-LC3, as shown in Figure 5A, indicating that the seipin-induced abnormal morphology of the LDs was deglycosylation-dependent. Together with Figure 3, these results imply that the autophagy system was activated as an adaptive response when the mutant seipin was overexpressed in the cells.

\section{Discussion}

Previous studies have shown that seipin is one of the most important factors regulating the morphology of lipid droplets ${ }^{[7,12]}$. In yeasts and drosophila, seipin controls the size of LDs. The overexpression of seipin in Fld 1p (a functional homologue of human seipin)-deficient yeasts can rescue LD-associated defects, while in drosophila, the overexpression of seipin can downregulate lipogenesis in non-adipose tissues $^{[8,14]}$. However, in mammalian cells, the impact of glycosylation site mutations in human seipin on lipid droplets has seldom been studied. By using the co-transfection method and LDs-specific dye Nile Red in the present study, we showed clearly that lipid droplets became increasingly larger and fused in seipin mutant-transfected human cells (Figure $3 \mathrm{~A}$ and $3 \mathrm{~B}$ ), indicating that mutations in the glycosylation site of seipin played an important role in the morphology of LDs. Glycosylation is required for proper protein folding and normal function, whereas the deglycosylation of proteins appears to result in protein misfolding and dysfunction ${ }^{[5]}$.

Seipin is an ER-located membrane protein that contains a glycosylation site facing the ER lumen, and seipin interacts directly with the LDs in the cytoplasm ${ }^{[1,13]}$. Furthermore, previous researchers have reported that the autophagy com- ponent LC3 interacts directly with LDs and participates in the formation of $\operatorname{LDs}^{[11,16-19]}$. To further investigate whether the mutation of the seipin glycosylation site was crucial to the activation of the autophagy system, we used the protein glycosylation-inhibition drug tunicamycin to mimic the deglycosylation of wildtype seipin. When wild-type seipin-transfected cells were pre-treated with tunicamycin and compared to mutant seipin-transfected cells, similar morphological and biochemical results appeared, such as large vacuoles in the cytoplasm, an identical pattern of the cleaved bands of the $\mathrm{N}$-terminal fragment of seipin, and an equivalent height to the protein band of full length seipin (Figure 5A, Figure 6A, and Figure $5 \mathrm{C}$ ). These data indicate that physiologically normal seipin functioned as a potent inhibitor of $\operatorname{LD}_{\text {fusion }}{ }^{[12,14]}$, and autophagy was activated as an adaptive response to engulf and break down the giant or "supersized" LDs to balance the LDs and lipid metabolism within the cells ${ }^{[11]}$. Moreover, these results imply that the induction of abnormal lipid droplets and the subsequent activation of autophagy is also deglycosylation-dependent.

Given that previous studies reported that the autophagy component LC3 is involved in the formation of LDs and that the autophagy system is required for LD breakdown ${ }^{[11,16-19]}$, we investigated whether seipin had a direct relationship to the autophagy system. We showed that both wild-type and mutant seipin co-localized with GFP-LC3 (Figure 1), indicating that there is a direct relationship between seipin and the autophagy system. Additionally, our results indicate that the co-expression of mutant seipin (N88S or S90L) with the autophagosome marker GFP-LC3 in HEK-293, H1299, and MES23.5 cells influenced the distribution of GFP-LC3 and resulted in the formation of giant vacuoles in the cytoplasm; in addition, overexpressed mutant seipin affected the autophagy system by disrupting normal LD metabolism (Figure 2, Figure 3). Furthermore, under the condition of autophagy inhibition, as shown in Figure 4A, mutant seipin-induced abnormal vacuoles fused into larger vacuoles. Together with Figure 2 and Figure 3, our data imply that mutant seipin leads to abnormal morphological changes in LDs, causing the formation of large vacuoles mediated by the autophagy component LC3, whereas the inhibition of autophagy impairs LC3's adaptive response to degrade abnormal LDs.

Although previous studies have reported that $\mathrm{dHMH}-\mathrm{V}$ is caused by toxic cellular inclusions formed in the ER due to misfolded mutant seipin (N88S or S90L) ${ }^{[1,5,6,20]}$, there is a lack of evidence showing how mutant seipin impacts the autophagy system. In this report, we demonstrated that the autophagy system was only activated in cells transfected with mutant versus wild-type seipin (Figure 6A and 6C). In addition, the levels of the ER-stress indicator Bip were found to be elevated in mutant seipin-transfected cells (Figure 6D), which implies that autophagy activation might be downstream of mutant seipin-induced ER-stress, which is consistent with previous studies showing that aggregates of another misfolded protein, PolyQ, can activate the autophagy system by triggering ERstress $^{[22]}$. 
Taken together, the mutations in seipin disrupted its normal physiological function in regulating lipid droplet metabolism. Subsequently, autophagy was enacted as an adaptive response to break down abnormal LDs, and the interruption of autophagy accelerated the fusion of abnormal LDs. The activation of the autophagy system as well as the abnormal morphology of the LDs caused by mutant seipin was deglycosylationdependent, implying a close relationship between mutant seipin, the autophagy system and LD morphology. Moreover, mutations of seipin at its glycosylation site lead to protein misfolding in the ER, causing ER stress and subsequent activation of the autophagy system. One possible explanation for the co-occurrence of lipodystrophy and motor neuropathy might be related to the accumulation or loss of lipids in motoneurons and adipocytes. For example, the loss of the lipin 1 gene (involved in lipid metabolism and adipogenesis) in Schwann cells in mice was found to result in lipid loss and nerve dysfunction $^{[4]}$. Thus, the loss of the normal function (caused by mutations) of seipin might be a key factor in the disease progression of lipodystrophy and motor neuropathy, and the autophagy system therefore represents a cellular target for abnormalities in lipid metabolism.

\section{Acknowledgements}

This work was supported by the National Basic Research Program of China (No 2014CB932002), the CAS Strategic Priority Research Program (№ XDB14030502), the CAS/SAFEA International Partnership Program for Creative Research Teams, and the National Natural Science Foundation of China (№ 81273004, 31470829, 20977093, and 10935009). We thank Prof Daisuke ITO from Keio University (Japan) for providing the seipin plasmids.

\section{Author contribution}

Hua-dong FAN, Li-jun WU designed the study; Hua-dong FAN, Yu-xiang SUN, and Shao-hai XU performed the research; Hua-dong FAN, and Li-jun WU analyzed the data; Hua-dong FAN, Shao-peng CHEN, and Li-jun WU wrote the paper.

\section{References}

1 Ito D, Suzuki N. Seipinopathy: a novel endoplasmic reticulum stressassociated disease. Brain 2008; 132: 8-15.

2 Chen W, Yechoor VK, Chang BH, Li MV, March KL, Chan L. The human lipodystrophy gene product Berardinelli-Seip congenital lipodystrophy $2 /$ seipin plays a key role in adipocyte differentiation. Endocrinology 2009; 150: 4552-61.

3 Cui X, Wang Y, Tang Y, Liu Y, Zhao L, Deng J, et al. Seipin ablation in mice results in severe generalized lipodystrophy. Human Mol Genet 2011; 20: 3022-30.
4 Agarwal AK, Garg A. Seipin: a mysterious protein. Trends Mol Med 2004; 10: 440-4.

5 Windpassinger C, Auer-Grumbach M, Irobi J, Patel H, Petek E, Hörl G, et al. Heterozygous missense mutations in BSCL2 are associated with distal hereditary motor neuropathy and Silver syndrome. Nat Genet 2004; 36: 271-6.

6 Ito D, Fujisawa T, lida H, Suzuki N. Characterization of seipin/BSCL2, a protein associated with spastic paraplegia 17. Neurobiol Dis 2008; 31: $266-77$

7 Fei W, Du X, Yang H. Seipin, adipogenesis and lipid droplets. Trends Endocrinol Metab 2011; 22: 204-10.

8 Ashrafi K, Tian Y, Bi J, Shui G, Liu Z, Xiang Y, et al. Tissue-autonomous function of Drosophila Seipin in preventing ectopic lipid Droplet formation. PLoS Genet 2011; 7: e1001364.

9 Martin S, Parton RG. Lipid droplets: a unified view of a dynamic organelle. Nat Rev Mol Cell Biol 2006; 7: 373-8.

10 Farese RV Jr, Walther TC. Lipid droplets finally get a little R-E-S-P-EC-T. Cell 2009; 139: 855-60.

11 Singh R, Kaushik S, Wang Y, Xiang Y, Novak I, Komatsu M, et al. Autophagy regulates lipid metabolism. Nature 2009; 458: 1131-5.

12 Yang H, Galea A, Sytnyk V, Crossley M. Controlling the size of lipid droplets: lipid and protein factors. Curr Opin Cell Biol 2012; 24: 50916.

13 Binns D, Lee S, Hilton CL, Jiang QX, Goodman JM. Seipin is a discrete homooligomer. Biochemistry 2010; 49: 10747-55.

14 Fei W, Shui G, Gaeta B, Du X, Kuerschner L, Li P, et al. Fld1p, a functional homologue of human seipin, regulates the size of lipid droplets in yeast. J Cell Biol 2008; 180: 473-82.

15 Fei W, Li H, Shui G, Kapterian TS, Bielby C, Du X, et al. Molecular characterization of seipin and its mutants: implications for seipin in triacylglycerol synthesis. J Lipid Res 2011; 52: 2136-47.

16 Singh R. Autophagy and regulation of lipid metabolism. Results Probl Cell Differ 2010; 52: 35-46.

17 Shibata M, Yoshimura K, Tamura H, Ueno T, Nishimura T, Inoue T, et al. LC3, a microtubule-associated protein $1 \mathrm{~A} / \mathrm{B}$ light chain3, is involved in cytoplasmic lipid droplet formation. Biochem Biophys Res Commun 2010; 393: 274-9.

18 Shibata M, Yoshimura K, Furuya N, Koike M, Ueno T, Komatsu M, et al. The MAP1-LC3 conjugation system is involved in lipid droplet formation. Biochem Biophys Res Commun 2009; 382: 419-23.

19 Dong H, Czaja MJ. Regulation of lipid droplets by autophagy. Trends Endocrinol Metab 2011; 22: 234-40.

20 Yagi T, Ito D, Nihei Y, Ishihara T, Suzuki N. N88S seipin mutant transgenic mice develop features of seipinopathy/BSCL2-related motor neuron disease via endoplasmic reticulum stress. Human Mol Genet 2011; 20: 3831-40.

21 Ashrafi K, Fei W, Shui G, Zhang Y, Krahmer N, Ferguson C, et al. A role for phosphatidic acid in the formation of "Supersized" lipid Droplets. PLoS Genet 2011; 7: e1002201.

22 Kouroku Y, Fujita E, Tanida I, Ueno T, Isoai A, Kumagai H, et al. ER stress (PERK/elF2alpha phosphorylation) mediates the polyglutamineinduced LC3 conversion, an essential step for autophagy formation. Cell Death Differ 2007; 14: 230-9. 